\title{
The Short-Period Binary Frequency Among Low-Mass Pre-Main Sequence Stars
}

\author{
ROBERT D. MATHIEU \\ Department of Astronomy, University of Wisconsin, \\ Madison, WI 53706, USA
}

\section{INTRODUCTION}

The pre-main sequence (PMS) binary frequency is a fundamental datum in the study of binary formation. It reflects on numerous basic issues, such as:

- The formation process. Binary stars are the primary branch of the star-formation process, and thus their frequency is an essential challenge to star-formation theories. (Indeed, the infrequency of single-star formation is likely as significant as the binary frequency.)

- The epoch of binary formation. Assessing whether the binary population exists in total by the pre-main sequence phase sets an upper limit on the binary formation timescale.

- Early period evolution. The frequency distribution as a function of period of PMS binaries, when compared to the distribution at the zeroage main sequence, can shed light on early orbital evolution.

- The interaction of binaries with disks. The formation and consequent dynamical evolution of a binary with semi-major axis less than typical disk radii must substantially modify disk structures and accretion flows. Thus the binary frequency might differ between PMS stars with and without associated disks.

The theme of this meeting, to combine complementary methods of binary research, is particularly appropriate for the youngest binaries; to address the above questions will require knowledge of the binary frequency at all periods. This paper will consider the frequency of "short-period" binaries, which we define here as binaries with periods of less than 100 days. We define the shortperiod binary frequency as the number of such binaries divided by the number of distinct bound systems (counting a single star as a system). Roughly speaking, this frequency addresses the question of how often a short-period binary is produced when a star-formation event occurs.

The short-period binary frequency may also be particularly valuable for the study of binary-disk interactions. It has long been suggested that low-mass stellar companions will drive annular gaps in disks (e.g., Lin \& Papaloizou 1992). Recently, Artymowicz et al. (1991) have further argued that comparable-mass components in near-circular orbits will act dynamically to restrict accretion from a circumbinary disk across the binary orbit. Thus circumstellar disks, continuing to accrete but insufficiently replenished, exhaust themselves leaving only a circumbinary disk. More dramatically, capture formation of a binary may entirely disrupt the circumbinary disk (Clarke, private communication), ultimately also leading to exhausted circumstellar disks. 
Thus it is plausible that the binary frequency might be smaller among PMS binaries with evidence for circumstellar disks. In fact, PMS stars with evidence for circumstellar disks have long been known to exist in wide binaries $\left(\mathrm{a}>\mathrm{r}_{\text {disk }} \approx 100 \mathrm{AU}\right)$. Similarly, but extending to smaller separations, Simon et al. (1992) do not find any difference in $\mathrm{H} \alpha$ emission between single stars and binaries with separations of greater than $1 \mathrm{AU}$. However, if circumstellar disks in binaries indeed disappear by starvation, the timescale of their exhaustion will depend, among other things, on the mass of the circumstellar disk and the accretion rate. The former will presumably be smaller for shorter period binaries. Consequently the lifetimes of circumstellar disks might be shortest in the shortest period binaries. A similar conclusion would also be drawn for any disruption mechanism which scales by the binary dynamical timescale. Thus, a reduced binary frequency among PMS stars with circumstellar disks might be most expected for the short-period binaries.

Because it is the most comprehensively available datum, in this paper we take $\mathrm{H} \alpha$ emission strength as an indirect diagnostic for circumstellar disks. Hartigan et al. (1990) have shown that $\mathrm{H} \alpha$ emission strength correlates with boundary layer emission, the latter presumed to be indicative of accretion rate at a stellar surface. In the disk paradigm, accretion at a stellar surface is fed by circumstellar disk material. Similarly, Skrutskie et al. (1990) find that $\mathrm{H} \alpha$ emission strength is correlated with $10 \mu \mathrm{m}$ emission and conclude that $\mathrm{H} \alpha$ emission is directly linked to the reservoir of material in inner disks. Thus strong $\mathrm{H} \alpha$ emission is arguably indicative of at least one circumstellar disk in a binary system $^{1}$. Note that $\mathrm{H} \alpha$ emission arises on size scales of a few stellar radii, much smaller than the separations of most binaries. Thus any influence of binaries on $\mathrm{H} \alpha$ emission would be indirect through their influence on the inner disk region.

Strom et al. (1989) suggested an $\mathrm{H} \alpha$ equivalent width of $10 \AA$ as a reasonable division of PMS stars with and without substantial accretion activity. We adopt that division here, and refer to the weaker lined stars as "weak-lined $T$ Tauri stars" (WTTS) and the stronger lined stars as "classical T Tauri stars" (CTTS). (Note that the naked T Tauri stars (e.g., Walter et al. 1988) are a subset of the WTTS.) We first discuss the short-period binary frequency among the WTTS and then among the CTTS. We then briefly consider the short-period binary frequency as a function of star-formation region. The short-period binary frequency among the entire sample of PMS stars is indistinguishable from that found among field solar-mass main-sequence (MS) stars, although the data show hints of deviations from the MS short-period binary frequency as functions of the presence of disks or site of formation.

\section{SHORT-PERIOD BINARY FREQUENCY: WEAK-LINED T TAURI STARS}

The first PMS spectroscopic binary, V826 Tau, was found in a sample of five X-ray selected WTTS (Mundt et al. 1983), and since that time such stars have proven fruitful hunting grounds for PMS spectroscopic binaries. To date, one

\footnotetext{
${ }^{2}$ Photometry at $K$ exists for some binaries discussed below. In all but one case the $K$ fluxes corroborate $\mathrm{H} \alpha$ emission as a diagnostic for circumstellar disks.
} 
systematic radial-velocity survey of a large, reasonably homogeneous sample of X-ray WTTS has been completed. Mathieu et al. (1989; MWM) obtained multiple high-precision radial-velocity measurements over a timespan of at least a year for 53 WTTS in the Taurus-Auriga, Ophiuchus-Scorpius, and Corona Australis star-forming regions. They found five binaries for which they were able to obtain orbit solutions, in addition to the previously determined orbit for V826 Tau. They also discovered one certain long-period binary of unknown period and four additional binary candidates. Restricting their analysis to periods of less than 100 days for completeness, they found a binary frequency of $9 \% \pm 4 \%$. They concluded that this frequency was consistent with the MS binary frequency of $12 \% \pm 3 \%$ [as derived from Abt \& Levy (1976), modified according to Morbey \& Griffin (1987) and Abt (1987)].

The latest measurements presented in MWM were obtained in the summer of 1988. Observations have continued since then, albeit at a reduced rate, so that an update is worthwhile here. Progress has been made primarily in the Ophiuchus-Scorpius and Corona Australis star-forming regions. At the time of MWM, only two radial-velocity measurements were available for the majority of the stars observed in these regions. Today $70 \%$ of the original sample has five or more radial-velocity measurements over an interval greater than 1000 days, and no star has less than three observations. No additional stars have been found to have velocity amplitudes greater than $4 \mathrm{~km} \mathrm{~s}^{-1}$ (the criterion for binary detection of MWM), although a few lower amplitude variables have been found. Two additional stars have been added to the Corona Australis sample, neither of which are velocity variable. The phase coverage for the binary 162819-2423S, which was limited in MWM, has been substantially improved, with no significant change in the derived orbital elements. A new orbit determination has been made for one of the binary candidates, 155809-2219, with a period of 16.9 days. This system is a spectroscopic triple, with the binary primary and a tertiary evident in the spectra. The number of survey radial-velocity measurements in the Taurus-Auriga region has not been as significantly increased. Nonetheless, at present all stars save one have been observed at least three times over a timespan of roughly 700 days. Despite the expressed optimism of MWM, additional observations have not produced orbit solutions for the remaining three candidate binaries, V827 Tau, V836 Tau and 042916+1751.

Observations of the MS binary population have also progressed since MWM. In particular, Duquennoy \& Mayor (1991) have published their study of mainsequence solar-mass stars in the solar neighborhood. Among 164 systems they find 13 binaries with periods less than 100 days. One of these is a W UMa star which they did not detect via radial-velocity observations, so for the purposes here their results give a frequency of short-period binaries of $12 / 164$ or $7 \% \pm 2 \%$. The difference from the corrected Abt \& Levy short-period binary frequency of $12 \%$ is curious. Two thirds of the Abt \& Levy stars are also in the Duquennoy and Mayor sample, but only one quarter of the Abt \& Levy short-period binaries are among these overlapping systems. If the samples of both studies were combined, the MS frequency of short-period binaries would be $11 \% \pm 2 \%$. The pedigree of the Abt and Levy short-period orbit determinations is high (all were confirmations of earlier orbits) and none violate the strictures of the Branch effect (Abt 1987). The difference in short-period frequency may be due to some other selection effect, but this remains to be shown. Here we will use 
$7 \%$ as the MS short-period binary frequency, but the reader is cautioned that first, this may be an underestimate and second, there is a $30 \%$ sampling error in this value.

Now, adding the two new stars in the Corona Australis region to the sample and 155809-2219 to the binary count, we find a binary frequency among these WTTS of $6 / 55$ or $11 \% \pm 4 \%$. For a MS short-period binary frequency of $7 \%$ the expected number of short-period binaries among these WTTS is 4 , so the conclusion remains that the short-period binary frequency among the WTTS is indistinguishable from that in the field.

There have been intriguing reports at this meeting of longer-period PMS binary frequencies higher than found among MS binaries. Due to the large sampling error, these spectroscopic results cannot distinguish such higher frequencies for short-period PMS binaries. For example, a factor of two excess in the frequency of short-period PMS binaries compared to MS binaries would predict the number of short-period binaries in the MWM sample to be 8 , as compared to the 6 actually detected.

\section{SHORT-PERIOD BINARY FREQUENCY: CLASSICAL T TAURI STARS}

The spectral peculiarities of $T$ Tau were noted over four decades ago, and $T$ Tauri stars have since been intensively studied at high spectral resolutions. Thus it is curious that few spectroscopic binaries have been found among the CTTS. Indeed at present the count of spectroscopic binaries with orbit determinations among CTTS remains at most three, the status of two binaries as CTTS being uncertain. The three cases are:

V4046 Sgr - This double-lined binary has a circular orbit with a period of 2.4 days (de la Reza et al. 1986). It has an emission-line spectrum, strong lithium lines and large near- and far-infrared excesses. Its status as a CTTS is ambiguous only because it is one of the few T Tauri-like stars isolated from molecular emission, dark clouds or evidence for recent star formation. Since its distance is unknown, its pre-main sequence status is unproven. Similarly, the source of the circumstellar material is uncertain. Herbig (1978) suggested as one of several possibilities that V4046 Sgr is an example of an interacting binary during the T Tauri stage of evolution.

AK Sco - This star is a double-lined binary with a period of 13.3 days (Andersen et al. 1989). Its status as a CTTS has also been uncertain. While $\mathrm{H} \alpha$ has been observed in emission (Herbig \& Bell 1988), other spectra show $\mathrm{H} \alpha$ in absorption and no emission lines have been found in the blue (Andersen et al. 1989). During this meeting, E. Jensen monitored the $\mathrm{H} \alpha$ line for one orbital period at intermediate spectral resolution (fiber-optic echelle spectrograph at Kitt Peak) and found a variable, broad emission line with a strong central reversal. The $\mathrm{H} \alpha$ equivalent width was only a few $\AA$, so by strict definition the star is not a CTTS. However AK Sco has a hotter photosphere $(6500 \mathrm{~K})$ than typical for CTTS, so arguably the emission flux is sufficient to merit classification as a CTTS. Equally importantly, AK Sco shows excess flux throughout the infrared wavelengths indicative of extended material. 
GW Ori - Mathieu and Latham obtained an orbit for GW Ori with a period of 242 days (Mathieu et al. 1991). GW Ori is a classical T Tauri star in all respects, including strong $\mathrm{H} \alpha$ emission and very large infrared excesses.

These anecdotal cases are extremely important for establishing whether the CTTS environment can exist in short-period binaries, and as case studies for analysis of the interactions of binaries and disks (e.g., Mathieu et al. 1991, Mathieu 1992). In this regard it is worth noting that age estimates for these binaries are $10^{6} \mathrm{yr}$ or greater and thus they are not remarkably young. In any case, since they were not discovered in systematic surveys, these binaries provide little quantitative information on the binary frequency among CTTS.

Various authors have suggested velocity variability for other CTTS, although in none of these cases does the number of observations suffice to prove the variability periodic or to show systematic velocity variation indicative of orbital motion. The first systematic radial-velocity study of $T$ Tauri stars with sufficient precision to address the question of binarity was made by Herbig (1977). While typically he obtained only a few radial-velocity measurements per star, Herbig made the statistical argument that the number of velocity measurements significantly deviating from the mean radial velocity of the Taurus-Auriga association was consistent with that expected if the T Tauri binary population were similar to the MS binary population as defined by Abt \& Levy (1976).

More recently, a radial-velocity study of 44 stars in the Taurus-Auriga star-forming region has been done at the Harvard-Smithsonian Center for Astrophysics. The sample included $\mathrm{H} \alpha$-selected WTTS and CTTS. Initially the spectra were obtained for a study of rotation among T Tauri stars (Hartmann et al. 1986, to which the reader is referred for technical details). The observing program has been continued by the author and Dr. Hartmann, with the most recent observations concentrated on candidate velocity variables. Here we present a preliminary analysis of these data.

An accurate assessment of the radial-velocity measurement errors is critical to detecting real velocity variability. This is particularly difficult for CTTS, each of which has a unique spectrum. Even at similar signal-to-noise levels the quality of velocity measurement can vary significantly between CTTS due to line emission, veiling and rotation. In the analysis here we have only included highquality radial-velocity measurements, at the expense of excluding numerous spectra and a few stars from the analyses. Thus the final sample is somewhat biased toward less active $T$ Tauri stars, and excludes stars with $v \sin i>50$ $\mathrm{km} \mathrm{s}^{-1}$. Specifically, the sample includes 25 CTTS and 11 WTTS. The median number of observations per star is five, and most have been observed at least once in each of at least three years. Nonetheless, a few have provided good velocity measurements in only two seasons. Based on typical measurement repeatability we estimate velocity measurement errors of less than $2 \mathrm{~km} \mathrm{~s}^{-1}$.

Several stars in the sample show low-amplitude variability in their measured radial velocities, but as yet the data are insufficient to assure orbital motion. A few merit discussion here:

CW Tau - This CTTS has been noted previously as a candidate spectroscopic binary. Herbig (1977) measured a velocity of $28 \mathrm{~km} \mathrm{~s}^{-1}\left(12 \mathrm{~km} \mathrm{~s}^{-1}\right.$ greater than the molecular line velocity along the same line of sight) while Edwards et 
al. (1987) measured a velocity of $10.7 \mathrm{~km} \mathrm{~s}^{-1}$. The CfA observations provide two velocity measurements differing by $10 \mathrm{~km} \mathrm{~s}^{-1}$. In fact eight spectra of this star have been obtained, but in most the emission and veiling were sufficiently pronounced that only poor or no velocity measurements could be made.

DQ Tau - Several of the numerous spectra produced two blended correlation peaks, with a maximum observed velocity separation of $\sim 20 \mathrm{~km} \mathrm{~s}^{-1}$. The remaining spectra produced single correlation peaks of variable width. This CTTS is thus a candidate double-lined system of low velocity amplitude, which we continue to monitor. In two instances the correlation spectra evolve from two blended narrow peaks to a single broad peak to a single narrow peak in less than six days, suggesting a short-period binary despite the low velocity amplitude.

Lk H $\alpha$ 266S, UX Tau A - These stars provide velocity measurements of good precision and show velocity variation larger than expected from measurement error. UX Tau A, a WTTS by $\mathrm{H} \alpha$ emission, has large infrared excesses (Skrutskie et al. 1990).

RW Aur, UY A ur - RW Aur was tentatively suggested to be a spectroscopic binary by Hartmann et al. (1986). With the inclusion of recently obtained data the peak-to-peak range of the velocity measurements is $16 \mathrm{~km} \mathrm{~s}^{-1}$. However, RW Auriga has a highly variable spectrum which is typically heavily veiled; only a small fraction of the spectra produced velocity measurements satisfying the quality criterion here. Thus, Hartmann \& Stauffer (1989) concluded that the apparent velocity variability is more likely due to effects on line profiles caused by strong veiling. UY Aur has a similarly variable spectrum and only a few spectra provided velocity measurements, although seemingly of high quality.

Notably, none of these stars shows very high amplitude velocity variation. Other than RW Aur the largest observed peak-to-peak velocity variations among the single-lined binary candidates are $\sim 10 \mathrm{~km} \mathrm{~s}^{-1}$. The observed velocity separations of the components in the candidate double-lined binary DQ Tau are $\sim 20 \mathrm{~km} \mathrm{~s}^{-1}$. This lack of higher amplitude velocity variation suggests the absence in this sample of the shortest period binaries. Such an absence would be particularly intriguing for the CTTS in the context of disk-binary interactions, as discussed in the Introduction.

However, a sufficiently precise measure of the frequency of short-period binaries among the CTTS is hampered by small numbers. A quick estimate of the expected number of high-amplitude velocity variables can be made from the MWM results for the WTTS. Six binaries in the MWM sample showed peak-to-peak velocity excursions greater than $20 \mathrm{~km} \mathrm{~s}^{-1}$ within their first four observations. Given the respective sample sizes, only 3 stars among the CTTS would be expected to show variations in their velocities of similar magnitude. In fact one such star, DQ Tau, has been found.

A more formal analysis can be done. Following Herbig (1977), the distribution of measured radial velocities can be used to test the most straightforward hypothesis, to wit that the short-period binary population among the CTTS is the same as that of MS stars. We have taken a Monte Carlo approach, modeling the MS binary population based on the results of Duquennoy \& Mayor (1991) and taking the secondary mass function of Mazeh et al. (this meeting) 
for $\mathcal{M}_{2}>0.1 \mathcal{M}_{\odot}$. Given these specifications of the binary population (and assuming a $2 \mathrm{~km} \mathrm{~s}^{-1}$ measurement error), we numerically create a large sample of binaries and "observe" each with a pattern in time typical to those of the CfA observations. We then characterize each binary by the standard deviation of its measurements and examine the frequency of binaries with standard deviations above a certain limit. Initially we choose this limit to be $\sigma>10 \mathrm{~km} \mathrm{~s}^{-1}$ in order to focus on the frequency of short-period binaries, since we find that $70 \%$ of binaries with periods less than 100 days produce standard deviations greater than $10 \mathrm{~km} \mathrm{~s}^{-1}$ and that $80 \%$ of binaries with standard deviations greater than $10 \mathrm{~km} \mathrm{~s}^{-1}$ are binaries with periods less than 100 days.

The essential result is similar to that found from consideration of the WTTS: the predicted number of high-amplitude velocity variables is small. The expected number of velocity variables with $\sigma>10 \mathrm{~km} \mathrm{~s}^{-1}$ in the CTTS sample is 1.5. This number is similar to that found by simply counting the stars with $\sigma>$ $10 \mathrm{~km} \mathrm{~s}^{-1}$ in the more intensively observed Duquennoy and Mayor sample; the observed frequency there predicts 2.1 such stars in the CTTS sample. A similar Monte Carlo experiment using the corrected Abt and Levy binary population predicted 2.2 high-amplitude velocity variables among these CTTS. In fact, no stars with velocity variations of this magnitude have been observed among the Taurus-Auriga CTTS. Clearly, though, the expected values are sufficiently small that this null result does not suffice to prove a lower frequency of short-period binaries among the CTTS compared to the MS binary population.

In order to improve the sampling statistics somewhat, we lowered the detection criterion to $\sigma>3 \mathrm{~km} \mathrm{~s}^{-1}$. At this level the simulations show that binary detection is nearly complete to periods of 1000 days and sensitive to periods as long as 10,000 days. However, false detections due to measurement errors are no longer negligible. Applying this detection criterion, the Monte Carlo simulation predicts 5 detections, 3 of which are binaries. Among the CTTS we find 3 stars with $\sigma>3 \mathrm{~km} \mathrm{~s}^{-1}$ : CW Tau, RW Aur, and UY Aur. The candidate DQ Tau might also be included in the count, given that each component shows peakto-peak velocity excursions $\sim 20 \mathrm{~km} \mathrm{~s}^{-1}$. Taken straightforwardly there is again no evidence for a distinction of the CTTS and MS binary frequencies in this extended range of periods. Note though that the three single-lined candidate binaries are all cases with significant emission and veiling and thus less certain measurement precision, adding some ambiguity to this conclusion.

Thus at present the observed velocity distributions of the Taurus-Auriga CTTS cannot be distinguished from those expected of a MS binary population. While it remains true that no short-period binaries have been definitively detected among these CTTS, a statistically significant deficiency of such binaries is not established. A secure comparison of the CTTS and MS binary frequencies will require systematic radial-velocity studies of CTTS samples with at least twice the number of this Taurus-Auriga study. In this regard, radial-velocity surveys of CTTS in the $\lambda$ Orionis and NGC 2264 star-forming regions have also been done by Mathieu and Latham, including a sample of CTTS comparable in number to the Taurus-Auriga study. It remains intriguing that no short-period binaries have been definitively detected (GW Orionis having a period longer than 100 days), but a careful analysis of those data has not yet been completed.

Under the hypothesis that the CTTS short-period binary frequency does not differ from the WTTS frequency, we may analyze the union of the MWM 
survey and this Taurus-Auriga survey. Together they provide 91 PMS stars. The frequency of known short-period binaries in this sample is $6 / 91$ or $7 \% \pm 3 \%$, clearly consistent with the MS binary frequency of $7 \%$. The observed frequency is likely a lower limit to the true frequency, given the spectroscopic binary candidates of as yet unknown periods. However, the discovery of a few additional short-period binaries will not change the conclusion that the PMS short-period binary frequency is indistinguishable from the field. On the other hand, the present data cannot distinguish a small excess in the PMS binary frequency over that in the field. An excess of a factor two would predict 13 short-period binaries among these 91 stars; the 6 presently known represent only a $2 \sigma$ deviation from the hypothesis.

\section{SHORT-PERIOD BINARY FREQUENCY: STAR-FORMATION SITES}

MWM noted that the frequency of short-period binaries among the X-ray selected WTTS in the Taurus-Auriga region was curiously low compared to the Ophiuchus-Scorpius region. Similarly, the $11 \mathrm{H} \alpha$-selected WTTS in TaurusAuriga also have no evident short-period binaries. Combined, the TaurusAuriga WTTS sample numbers 37 stars, among which 2-3 short-period binaries would be expected given the MS frequency; one is known, V826 Tau. If the binary frequency is presumed independent of $\mathrm{H} \alpha$ emission strength, then the WTTS and CTTS samples in Taurus-Auriga can be combined to produce a sample of 62 PMS stars. The MS binary frequency would predict 4-5 short-period binaries in this sample; alternatively our Monte Carlo simulations would predict 4 stars with $\sigma>10 \mathrm{~km} \mathrm{~s}^{-1}$. V826 Tau remains the lone star satisfying either measure, although with respect to short-period binary frequency other cases with low velocity amplitude may yet be found among the binary candidates.

On the other hand, among the Ophiuchus-Scorpius sample of 24 stars, only 2 short-period binaries are expected given a MS binary frequency, while 5 have been found.

As just noted, the binary frequency derived from the union of the two regions is consistent with the MS binary frequency. However, combining the samples in this way is predicated on the PMS binary population being independent of star-formation site. Interestingly, if the Taurus-Auriga and Ophiuchus-Scorpius results are considered as two independent realizations of a MS-like parent population, a $\chi^{2}$ analysis shows the two experiments to exclude a universal PMS short-period binary frequency at the $98 \%$ confidence level. This marginal confidence level is somewhat sensitive to the adopted MS binary frequency as well as the periods of the binary candidates in Taurus-Auriga.

\section{CONCLUSIONS}

Repeated radial-velocity measurements have been obtained for 91 pre-main sequence stars. These measurements are the result of two surveys, one of $55 \mathrm{X}$-ray selected stars in the Taurus-Auriga, Ophiuchus-Scorpius, and Corona Australis star-forming regions and a second of $36 \mathrm{H} \alpha$ selected stars in the Taurus-Auriga association. In the X-ray selected sample, consisting entirely of weak-lined 
$\mathrm{T}$ Tauri stars, the short-period $(\mathrm{P}<100 \mathrm{~d})$ binary frequency is found to be $11 \% \pm 4 \%$, somewhat higher than but not distinguishable from the frequency of $7 \% \pm 2 \%$ found by Duquennoy \& Mayor (1991) among main-sequence solar-mass stars in the solar neighborhood.

The $\mathrm{H} \alpha$-selected sample consists of both weak-lined and classical $\mathrm{T}$ Tauri stars. Candidate spectroscopic binaries have been found but as yet no orbit determinations have been obtained. Among the subset of 25 classical $\mathrm{T}$ Tauri stars, no high-amplitude ( $\sigma>10 \mathrm{~km} \mathrm{~s}^{-1}$ ) velocity variables have been detected. This absence, and in general the paucity of known spectroscopic binaries among all CTTS, is intriguing in the context of close binaries restricting accretion and depleting circumstellar disks. However, Monte Carlo simulations show this sample size of CTTS to be too small to test this hypothesis at a statistically secure level. Also, this line of reasoning must still permit V4046 Sgr, AK Sco and GW Ori, all spectroscopic binaries with classical T Tauri characteristics.

The lack of large amplitude velocity variables among the classical $\mathrm{T}$ Tauri stars in Taurus-Auriga might alternatively be seen as a lower frequency of shortperiod binaries in the Taurus-Auriga region as a whole, since the weak-lined $\mathrm{T}$ Tauri stars in Taurus-Auriga also include few high-amplitude velocity variables. The entire Taurus-Auriga sample of 62 pre-main sequence stars has produced only one definitive short-period binary, compared to an expected number of 4-5 for a field binary population. In contrast, 5 short-period binaries have been found in a sample of 25 stars in the Ophiuchus-Scorpius region. The different frequencies in the two star-forming regions may be a hint that the pre-main sequence short-period binary frequency is not independent of site of formation.

While these deviations of the pre-main sequence binary frequency from a field-like binary population are intriguing possibilities, they are not well established. The secure result is that the observed frequency of short-period binaries among the entire sample of 91 pre-main sequence stars is $7 \% \pm 3 \%$, with the true frequency likely somewhat higher. As such, the present data are consistent with the hypothesis that the short-period binary frequency among all pre-main sequence stars is similar to that found among field main-sequence solar-mass stars. Evidently short-period binary formation occurs on timescales of less than $10^{6} \mathrm{yr}$, a typical age for the pre-main sequence stars studied here.

I would like to gratefully acknowledge the support of National Science Foundation Grant AST8814986, the Presidential Young Investigator program and the Wisconsin Alumni Research Foundation. And my thanks, with L. Hartmann and P. Myers, to R. Davis, R. Hewett, E. Horine, D. Latham and J. Peters who have obtained and reduced many of the spectra for this program.

\section{REFERENCES}

Abt, H.A. 1987, ApJ, 317, 353

Abt, H.A. \& Levy, S.G., 1976, ApJS, 30, 273

Andersen, J., Lindgren, H., Hazen, M.L., \& Mayor, M. 1989, A\&A, 219, 142

Artymowicz, P., Clarke, C.J., Lubow, S.H., \& Pringle, J.E. 1991, ApJ, 370, L35

de la Reza, R., Quast, G., Torres, C.A.D., Mayor, M., Meylan, G., \& Llorente de Andres, F. 1986, in New Insights in Astrophysics, ESA SP263, p. 107

Duquennoy, A. \& Mayor, M. 1991, $A \& A, 248,485$ 
Edwards, S., Cabrit, S., Strom, S., Heyer, I., Strom, K., \& Anderson, E. 1987, ApJ, 321,473

Hartigan, P., Hartmann, L., Kenyon, S.J., Strom, S.E., \& Skrutskie, M.F. 1990, ApJ, 354, L25

Hartmann, L.W., Hewett, R., Stahler, S., \& Mathieu, R.D. 1986, ApJ, 309, 275

Hartmann, L.W. \& Stauffer, J.R. 1989, $A J, 97,873$

Herbig, G.H. 1977, $A p J, 214,747$

Herbig, G.H. 1978, in Problems of Physics and Evolution of the Universe, (Academy of Sciences in the Armenian SSR, Yervan), p. 171

Herbig, G.H. \& Bell, K.R. 1988, Lick Obs. Bulletin, No. 1111

Lin, D.N.C. \& Papaloizou, J.P.C. 1991, in Protostars and Planets III, edited by M.S. Matthews and E. Levy (University of Arizona Press, Tucson), in press

Mathieu, R.D. 1992, in Evolutionary Processes in Interacting Binary Stars, IAU Symposium 151, edited by Y. Kondo, R. Sistero, and R. Polidan (Kluwer, Dordrecht), in press

Mathieu, R.D., Adams, F.C., \& Latham, D.W. 1991, AJ, 101, 2184

Mathieu, R.D., Walter, F.M., \& Myers, P.C. 1989, $A J, 98,987$

Morbey, C.L. \& Griffin, R.F. 1987, ApJ, 317, 343

Mundt, R., Walter, F.M., Feigelson, E.D., Finkenzeller, U., Herbig, G.H., \& Odell, A.P. 1983, ApJ, 269, 229

Simon, M., Chen, W.P., Howell, R.R., Benson, J.A., \& Slowik, D. 1992, ApJ, 384, 212

Skrutskie, M.F., Dutkevitch, D., Strom, S.E., Edwards, S.E., \& Strom, K.M. 1990, AJ, 99,1187

Strom, K.M., Strom, S.E., Edwards, S., Cabrit, S., \& Skrutskie, M. 1989, AJ, 97, 1451

Walter, F.M., Brown, A., Mathieu, R.D., Myers, P.C., \& Vrba, F. 1988, AJ, 96, 297

\section{DISCUSSION}

Note on discussion: Many of the questions and comments refer to the binary GW Ori. The focus of this paper is more specific than the oral presentation, and GW Ori is not discussed here. The reader is referred to Mathieu et al. (1991) for detailed discussion of GW Ori and Mathieu (1992) for a more general discussion of disks in the binary environment.

FEKEL: I just want to remind the listeners that your results apply to late-type stars not $\mathrm{O}, \mathrm{B}$, or A stars.

HENRY: Can you make any correction between the mass of the secondary and the size of the gap in the system?

MATHIEU: I'm not expert in disk dynamics, but I believe that the size of the gap is not very sensitive to the secondary mass. Also, recall that for comparable mass components, the distribution of material is not axisymmetric around the primary. Thus the concept of a simple annular gap breaks down. In this same vein, the specific numbers produced by our simple model for GW Ori should not be given too much weight. The essential point is that the dip in the spectral energy distribution indicates structure in the disk on size scale comparable to the GW Ori semi-major axis.

SIMON: Can you exclude the possibility that the long wavelength excess in GW Ori is attributable to an IR-luminous tertiary rather than a disk? 
MATHIEU: No, the IR companions add a significant ambiguity to this line of study. I have asked Andrea Ghez to examine GW Ori for a tertiary at $2 \mu \mathrm{m}$, but, of course, high angular resolution observations at wavelengths longer than $10 \mu \mathrm{m}$ would more directly address the issue. I should also note that in Mathieu, Adams, \& Latham $(A J, 101,2184,1991)$, we discuss in some detail the possibility that the far IR excess is from a circumbinary envelope. The IR data cannot definitively distinguish between these models. Hopefully, submillimeter fluxes will clarify the situation.

CLARKE: If the gap edge really is at $\sim 3$ orbital radii, then you wouldn't expect any eccentricity driving - the resonances aren't strong enough out there. You might also want to invoke some non-dynamical process to sweep a hole of that size because it takes a Hubble time dynamically!

MATHIEU: Yes, indeed, Lubow \& Artymowicz (1992, Binaries as Tracers of Star Formation, eds. Duquennoy and Mayor, in press) recently made this point as well. As you say, if this is the explanation of the long-lived circular orbit, then we must explain the size of the gap, which is created by the same resonances. At this point, I wouldn't put too much wieght on the specific numbers, our model is a very simple one for a complex situation. As I said to Todd Henry, the more essential result is that gaps or other structures in disks driven by binaries are detectable in spectral energy distributions, and the spectral energy distributions of several spectroscopic binaries show such structure.

MAZEH: The agreement between the model of GW Ori and the data is very impressive. Can you comment on the different assumptions of the model?

MATHIEU: The disk model is that of Adams, Lada, \& Shu (ApJ, 326, $865,1988)$. For the purposes of modelling the optical-IR data of GW Ori, in essence the model is an optically thick, spatially thin disk. A power-law radial temperature distribution is adopted, with a fit exponent (for GR Ori, the resultant exponent requires an active disk.) The gap adds the free parameters of its inner and outer radii, and the surface density and temperature of the optically thin dust in the gap. For extensive discussion of the details, please see our paper. 